\title{
Téoros
}

Revue de recherche en tourisme

\section{Sustainable Transport - Problems and Solutions, William R. BLACK, The Guilford Press, 2010, 299 pages, ISBN : 1606234854}

\section{Alain A. Grenier}

Volume 32, numéro 2, 2013

URI : https://id.erudit.org/iderudit/1036603ar

DOI : https://doi.org/10.7202/1036603ar

Aller au sommaire du numéro

\section{Éditeur(s)}

Université du Québec à Montréal

ISSN

0712-8657 (imprimé)

1923-2705 (numérique)

Découvrir la revue

Citer ce compte rendu

Grenier, A. A. (2013). Compte rendu de [Sustainable Transport-Problems and

Solutions, William R. BLACK, The Guilford Press, 2010, 299 pages, ISBN :

1606234854]. Téoros, 32(2), 127-128. https://doi.org/10.7202/1036603ar d'utilisation que vous pouvez consulter en ligne.

https://apropos.erudit.org/fr/usagers/politique-dutilisation/ 


\title{
POUR EN LIRE PLUS
}

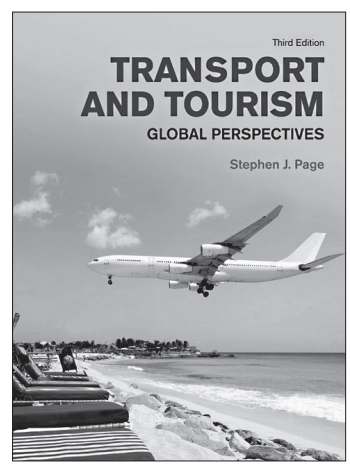

\author{
Transport and Tourism: global perspectives, $3^{\mathrm{e}}$ édition \\ Stephen PAGE \\ Pearson Education Canada, 2009, 480 pages \\ ISBN : 027371970X
}

Bien que le transport soit sans contredit la pierre angulaire du tourisme - il n'y a pas de voyage, même domestique, sans transport - la chose semble peu préoccuper les chercheurs. À preuve, les ouvrages sur la thématique sont rares. Pas surprenant que l'ouvrage de Stephen Page, autorité dans le domaine, en soit à sa troisième édition. Transport and Tourism: global perspectives propose une réflexion étoffée sur la relation parfois complexe quentretiennent le tourisme et l'offre de transport.

Louvrage comprend 10 chapitres organisés autour de quatre pôles (compréhension du transport à des fins touristiques - concepts et méthode d'analyse - l'analyse, l'utilisation et la disposition du transport - sa gestion et l'avenir). On y aborde des problématiques actuelles du transport touristique, le rôle des politiques gouvernementales de même que la règlementation du transport et sa privatisation. Deux chapitres sont respectivement consacrés à la demande et à l'offre en matière de transport touristique.

Cette $3^{e}$ édition s'attarde en plus sur les nouveaux défis du transport dans le contexte de l'évolution accélérée des transports au XXI siècle. Parmi ces défis, on note l'impact de la crise financière dans le secteur du transport aérien (aux États-Unis), les enjeux du transport à rabais, l'impact des changements climatiques sur le transport, le transport durable, le terrorisme et la criminalité et la sécurité. Le livre est particulièrement intéressant pour les étudiant(e)s et les gestionnaires puisqu'il ajoute, à la théorie, des exemples concrets de bonnes pratiques et de systèmes novateurs en matière de transport.

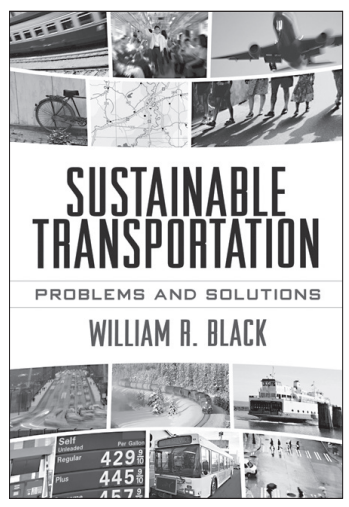

\section{Sustainable Transport - Problems and Solutions \\ William R. BLACK}

The Guilford Press, 2010, 299 pages

ISBN : 1606234854

Qui dit transport, de nos jours, doit aussi faire intervenir la question de l'empreinte écologique. William R. Black se penche justement sur la question du transport durable. À nouveau, louvrage sorganise autour de grands axes de réflexion : la nature du problème ( $1^{\mathrm{e}}$ partie), les solutions possibles ( $2^{\mathrm{e}}$ partie) et des conclusions ( $3^{\mathrm{e}}$ partie). Ce sont les 21 chapitres des deux premières parties qui retiennent notre attention. Le transport (actuel) y est abordé dans une perspective historique : comment avons-nous développé nos infrastructures? Il n’y a rien de tel que de revisiter les étapes de notre évolution pour mieux saisir les erreurs qui se sont accumulées. De là, Black ajoute à son analyse la prise de conscience environnementale et des nouveaux enjeux qu'elle soulève : la fin des réserves pétrolières, la qualité de l’air, la sécurité des véhicules, la congestion, etc.

Ce qui est particulièrement intéressant de cet ouvrage, est que la partie «solution» constitue plus des deux tiers de l'ouvrage. Les solutions proposées - des plus imaginatives aux plus radicales comme l'augmentation des coûts et des taxes sur la mobilité - sont décortiquées pour amener le lecteur à se construire un jugement informé et critique. Planification, urbanisme, politique de services et d'utilisation, éducation et sensibilisation, Black passe en revue chacun des grands aspects du transport sur lesquels les planificateurs et gestionnaires doivent se pencher, ensemble, afin de répondre, d'une part, aux besoins grandissants de mobilité des populations et d'apporter, d'autre part, des solutions durables aux impacts négatifs de la multiplication des véhicules et de la croissance du transport. À cet égard, quatre chapitres sont consacrés au rôle de la technologie de pointe dans les solutions à adopter : le rôle des télécommunications et du commerce électronique, les sources énergétiques alternatives, les nouveaux types de véhicules et les systèmes de transport intelligent.

On reprochera à ces deux ouvrages cependant (et du reste, à une majorité d'intellectuels du transport), d'oublier de traiter de la question des personnes aux prises avec des problèmes de mobilité, handicapées ou vieillissantes, pour qui le transport demeure une barrière majeure à 


\title{
POUR EN LIRE PLUS
}

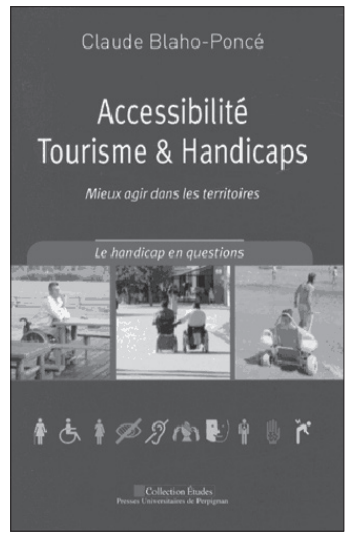

la pleine jouissance de la vie. Néanmoins, à une époque où la plupart des sociétés occidentales doivent renouveler leurs infrastructures en matière de transport, on souhaiterait que nos politicien(ne)s et gestionnaires prennent eux aussi le temps de lire ces ouvrages.

Alain A. GRENIER, Ph.D.

Département d'études urbaines et touristiques (ESG UQAM)

\author{
Accessibilité, tourisme \& handicaps - Mieux agir dans les territoires \\ Le handicap en questions \\ Claude BLAHO-PONCÉ \\ Presses de l'Université de Perpignan, 2013, 360 pages \\ ISBN : 978-2354121723
}

Le livre de Blaho-Poncé, Accessibilité Tourisme et Handicaps - Mieux agir dans les territoires, suscite une réflexion sur l'intégration, en amont des projets récréotouristiques, de la question de l'accessibilité des structures touristiques pour tous et sur une gouvernance qui tienne compte des besoins spécifiques des personnes handicapées. Issu de sa thèse de doctorat, l'ouvrage présente le cas du territoire du Languedoc Roussillon qui a su intégrer à son offre touristique existante, une offre destinée aux individus ayant un handicap. L'auteur soutient que les politiques touristiques locales sont à revoir en vue d'un développement durable et d'une accessibilité pour tous, le droit d'accès à une offre touristique étant fondamental. Cet ouvrage poursuit un triple objectif, celui d'étudier la réalité de l'accès des personnes en situation de handicap aux vacances et aux loisirs, celui d'examiner leurs attentes et besoins, et enfin celui d'observer l'application de la Loi française de février 2005 sur l'égalité des chances en région Languedoc Roussillon. Ce livre se veut être «[...] un ensemble de réflexions, de méthodes et d'outils pour mieux accompagner la réflexion des acteurs locaux» (Blaho-Poncé, 2012: 356).

Pour répondre à la question de base de cet ouvrage, «le tourisme-handicap peut-il être perçu par les professionnels du tourisme et par les élus comme un enjeu territorial? », l'auteur divise son livre en trois parties. La première, «Le handicap, un enjeu de société », tente d'apporter un éclairage nouveau sur la réalité de la condition des personnes vivant avec un handicap et d'explorer les représentations sociales liées à celui-ci, afin de dédramatiser l'accueil des personnes handicapées et de standardiser l'accès aux infrastructures touristiques. La seconde partie, «Pour un réel accès des personnes en situation de handicap aux vacances et aux loisirs ", justifie le droit aux vacances et aux loisirs pour chacun, y compris celui des personnes handicapées. Il fait également le point sur les actions, règlements et principes d'accessibilité à tout, pour tous. L'auteur relève, par ailleurs, certaines bonnes pratiques en termes d'accessibilité territoriale, entreprises par la région du Languedoc Roussillon. En troisième partie, «Accessibilité au tourisme et piste de développement pour entreprendre autrement», l'auteur présente des procédures méthodologiques et des outils d'évaluation pour une gestion de la destination qui conjugue les principes du développent durable et les standards d'accessibilité généralisée comme facteur de développement territorial.

L'intérêt premier du livre est la juxtaposition de la problématique de l'accessibilité des territoires touristiques pour tous, et plus particulièrement pour les personnes en situation de handicap, en lien avec les principes du développement durable. À la lecture de l'ouvrage, il parait tout à fait justifié de jumeler ces deux concepts. En effet, Blaho-Poncé souligne que «dans le cadre d'un tourisme respectueux de la sauvegarde et de la promotion de lenvironnemental social et humain, la possibilité pour les personnes en situation de handicap d'accéder aux vacances et aux loisirs dans l'offre généraliste, constitue un droit fondamental» (page 15).

Le livre Accessibilité Tourisme et Handicaps - Mieux agir dans les territoires est bien structuré, ses objectifs clairement définis et son développement, étayé d'exemples concrets, est facile à suivre. Cet ouvrage constitue certainement un apport intéressant dans le domaine du tourisme-handicap.

Thalie BOOGAERTS, étudiante Maîtrise en développement du tourisme

Département d'études urbaines et touristiques (ESG UQAM) 